\title{
Annonaceous Acetogenins
}

National Cancer Institute

\section{Source}

National Cancer Institute. Annonaceous Acetogenins. NCI Thesaurus. Code C118972.

A family of naturally occurring polyketides that consist of C32 or C34 long chain fatty acids and combined with a propan-2-ol unit at C-2 to form a gamma-lactone, which are isolated from various species of the plant family Annonaceae, with potential antineoplastic and antimicrobial activity. Annonaceous acetogenins bind to the ubiquinone catalytic site(s) within the mitochondrial NADH:ubiquinone oxidoreductase (complex I), and block the electron transport chain in mitochondria. In addition, the acetogenins bind to and block the activity of ubiquinone-linked NADH oxidase, an enzyme overexpressed in the plasma membranes of cancer cells. This inhibits adenosine triphosphate (ATP) production, decreases intracellular ATP levels, and induces tumor cell apoptosis. Compared to normal cells, cancer cells have higher ATP demands. The Annonaceous acetogenins also inhibit microbial glucose dehydrogenase 6 . 